\title{
Characteristics analysis of cerebral vascular angiography from transient ischemic attack accompanied with type 2 diabetes.
}

\author{
Dayong Du ${ }^{1 \#}$, Yanchao Shi ${ }^{2 \#}$, Hang Xü ${ }^{2}$, Heliang Zhang ${ }^{1}$, Zaiyu Guo ${ }^{\text {* }}$ \\ ${ }^{1}$ Department of Neurology, Tianjin Teda Hospital, Tianjin, 300457, PR China \\ ${ }^{2}$ Department of Neurology, Tianjin Port Hospital, Tianjin, 300456, PR China \\ \#These authors contributed equally.
}

\begin{abstract}
Objective: To explore the characteristics of cerebral vascular angiography from transient ischemic attack (TIA) accompanied with type 2 diabetes (T2D).

Methods: TIA patients with complete data in Tianjin Teda Hospital were retrospectively analyzed from December 2011 to November 2015 and 336 patients were taken up, which were divided into T2D group (152 cases) and control group (184 cases). By use of $C$ type arm angiography system, cerebral vascular stenotic degrees, determination of intracranial or extracranial artery, and the collateral compensative capacity were compared between two groups. Results: Cerebral vessel stenotic degree was significantly different between T2D group and the control group $\left(\chi^{2}=20.03, P<0.05\right)$. Patients with moderate to severe stenosis and occlusion were more in T2D group than those in the control group $\left(\chi^{2}=18.94, P<0.05\right)$. Control group was mainly with non-diffuse vascular lesions and compensatory form collateral vessels. In 3 subgroup, with the increase of glycosylated hemoglobin (unsatisfactory blood glucose control), the incidence rate of moderate and severe stenosis was increasing significantly $(P<\mathbf{0 . 0 5})$. Conclusion: Severe cerebral vascular stenosis is more easy to happen in patients of TIA accomplicated with T2D, which was distinguished by multiple vascular lesions, diffuse vascular lesions and less collateral compensative capacity. Unsatisfactory blood glucose control was a bad factor for these patients.
\end{abstract}

Keywords: Transient ischemic attack, Type 2 diabetes, Cerebral vascular angiography, Vascular disease, Case-control study.

Accepted on October 26, 2017

\section{Introduction}

Transient ischemic attack (TIA) was originally defined clinically by the temporary nature with less than $24 \mathrm{~h}$ of the associated neurologic symptoms, which is one of the common ischemic cerebro vascular disease [1]. In 2009, American Heart Association (AHA) and American Stroke Association (ASA) gave a new definition of TIA which was published in the Stroke "TIA is a transient episode of neurologic dysfunction caused by ischemia (loss of blood flow)-either focal brain, spinal cord, or retinal-without acute infarction (tissue death)" [2]. Previous studies have indicated that TIA patients had different degree of intracranial or extracranial artery stenosis, and the occurrence, development and turnover of TIA were related to the degree and nature of the arterial stenosis $[3,4]$. Epidemiological data showed that diabetes mellitus (DM) is one of the independent risk factor for stroke [5]. Especially patient with type 2 diabetes (T2D), the risk of stroke is $1.5-3$ times higher than that of the healthy human [6], which the main cause was cerebral infarction [7]. In addition, DM is also one of the important risk factors for intracranial artery atherosclerosis [8,9]. Intracranial and extracranial joint lesions were more in patients of TIA accomplicated with T2D [10].

Digital Subtraction Angiography (DSA) was set as the gold standard by imaging for evaluating the cerebral artery stenosis and occlusion $[11,12]$. It can not only determine the location and degree of stenosis, judge the nature of the plaque, but also can dynamically observe the blood flow, perfusion and the collateral compensative capacity $[13,14]$. By a retrospective designed method, we analyzed the aortocranial angiography from patients of TIA accomplicated with T2D who were detected by DSA, in order to realize the influence of T2D on cerebral artery blood vessel and provide scientific basis for secondary prevention of patients with TIA and T2D.

\section{Materials and Methods}

\section{Patients}

TIA patients with complete data in Tianjin Teda Hospital were retrospectively analyzed from December 2011 to November 2015 and 336 patients were taken up in this research. Before 
the surgery, comprehensive physical examination and systematic examination of the nervous system were done on patients.

Inclusion criteria were: 1) conform to the new definition of TIA in 2009 by AHA and ASA; 2) cases all checked by magnetic resonance imaging (MRI). Exclusion criteria were: 1) patients with type $1 \mathrm{DM} ; 2$ ) surgery of stenting for cerebral artery stenosis; 3) Intracranial occupying hemorrhage, intracerebral hemorrhage and/or subarachnoid hemorrhage; 4) without MRI; 5) comatose patients; 6) serious heart, lung, kidney, liver disease; 7) contrast agent allergy; 8) severe bleeding tendency; 9) other cerebral angiography; 10) patients or family members do not agree.

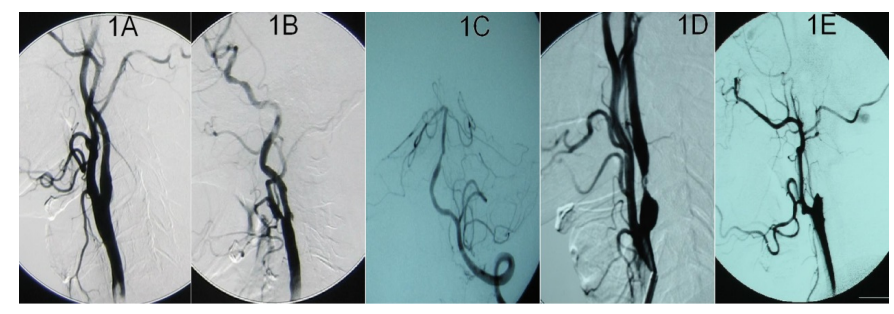

Figure 1. The degree of vascular stenosis; A) normal( $0 \%-29 \%)$, B) mild (30\%-49\%), C) moderate (50\%-69\%), D) severe (70\%-99\%), and E) occlusion (100\%).

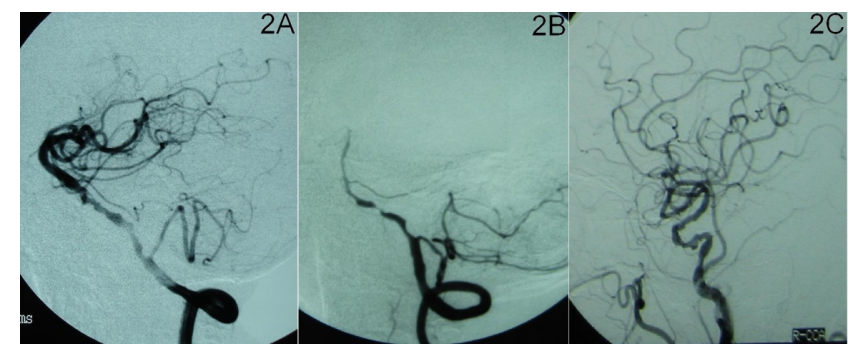

Figure 2. Diffuse vascular lesions; A) The length $\geq 20 \mathrm{~mm}, \mathrm{~B}) 1$ vessel with multiple lesions, $C$ ) The whole or most blood vessels were thin, stiff or obvious tortuous, in a loose shot like spring.

Patients with TIA were divided into T2D group (152 cases) and the control group (184 cases). T2D was diagnosed with 1999 WHO criteria (FPG $\geq 7.0 \mathrm{mmol} / \mathrm{L}$ and or $2 \mathrm{~h} \mathrm{PG} \geq 11.1$ $\mathrm{mmol} / \mathrm{L})[15]$. Blood of the patients was collected at the next morning after admission and was set to detect the glycosylated hemoglobin. Patients in T2D group were divided into 3 subgroups according to glycosylated haemoglobin $\leq 6.5 \%$ (T2D-1), 6.5\%-7.5\% (T2D-2), and $\geq 7.5 \%$ (T2D-3). The gentle, age, outbreak of TIA and hypertension in T2D and the control group showed no difference $(\mathrm{P}>0.05)$, whereas dysregulation of lipid metabolism was high in T2D group $(\mathrm{P}<0.05)$ (table 1). After the surgery, body temperature, pulse, respiration and blood pressure were monitored, and the consciousness, pupil and nervous system signs were observed. The patients can eat food after $6 \mathrm{~h}$, lie in bed for $24 \mathrm{~h}$, and puncture side of the lower limb should brake for $24 \mathrm{~h}$. The study and treatment methods were approved by the Institutional Ethics Committee of our hospital.

\section{Digital subtractive angiography (DSA)}

$\mathrm{C}$ type arm angiography system and non-ionic contrast agent (Omnipaque, 300 g/L, Ge pharmaceutical (Shanghai) co., LTD) were used. The right (or left) femoral artery was punctured by Seldinger technique. $0.89 \mathrm{~mm}$ hydrophilic guide wire was used to lead 5F angiography catheter to the near-end of aortic arch, bilateral common carotid artery, and bilateral subclavian arteries, respectively. Aortic arch and aortocranial angiography were done and magnification and multi angle angiography for suspicious lesions were prepared.

\section{Vessel stenotic degrees}

The evaluation of extracranial arterial stenosis was done according to the North American Symptomatic Carotid Endarterectomy Trial (NASCET) [16]. The degree=[(stenosis distal artery diameter-narrowest diameter)/stenosis distal artery diameter] $\times 100 \%$. The degree of intracranial arterial stenosis was evaluated by Warfarin-Aspirin Symptomatic Intracranial Disease (WASID). The degree $=(1$-narrowest diameter/diameter of the normal blood vessel of the same artery) $\times 100 \%$. The degree of vascular stenosis was divided into normal $(0 \%-29 \%$, Figure 1A), mild (30\%-49\%, Figure 1B), moderate (50\%-69\%, Figure 1C), severe (70\%-99\%, Figure 1D), and occlusion (100\%, Figure 1E) [17]. When the damage happened on more than 2 vessels, it was definited as multiple vessel lesions. Diffuse vascular lesions include: 1 ) the length $\geq 20 \mathrm{~mm}$ (Figure $2 \mathrm{~A}$ ); 2) 1 vessel with multiple lesions (Figure $2 \mathrm{~B}$ ); and 3 ) the whole or most blood vessels were thin, stiff or obvious tortuous, in a loose shot like spring (Figure 2C), which was set as severe stenosis. The judgment of display situation and stenosis degree in all blood vessels was completed by 2 experienced neuro-interventional physicians.

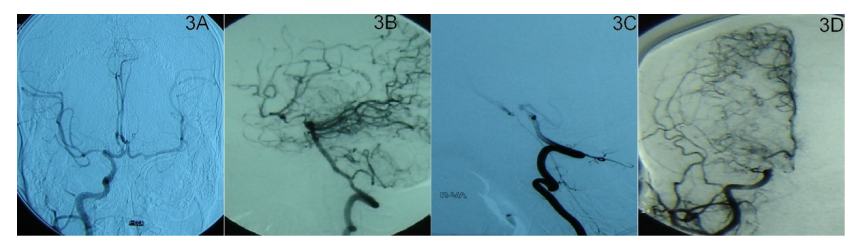

Figure 3. Collateral compensative capacity; A) Open standard of anterior communicating artery, B) Open standard of posterior communicating artery, $C \& D)$ Open standard of collateral circulation.

\section{Determination of intracranial artery and extracranial artery}

Extracranial artery include common carotid artery (CCA), extracranial internal carotid artery (E-ICA), extracranial vertebral artery (E-VA), and subclavian artery (SCA), whereas intracranial artery include intracranial internal carotid artery (IICA), middle cerebral artery (MCA), anterior cerebral artery (ACA), posterior cerebral artery (PCA), intracranial vertebral artery (I-VA), basilar artery (BA), superior cerebellar artery (SCEA), and anterior inferior cerebellar artery (AICA). Intracranial artery and extracranial artery were referred to as cerebral artery. 


\section{Collateral compensative capacity}

1) Open standard of anterior communicating artery: Arteriography of unilateral ICA can show contrast agent through anterior communicating artery developing the opposite anterior, middle arterial trunk and branch (Figure 3A); 2) Open standard of posterior communicating artery: Arteriography of vertebral artery can show contrast agent through posterior communicating artery developing the trunk and branches of the internal artery (Figure 3B); 3) Open standard of collateral circulation: contrast agent is retrograde from the end of an artery developing the trunk and branches of another artery (Figures 3C and 3D). According to the collateral blood flow grading standard, the location and time of collateral blood flowing to ischemic area could be divided into 0-4 level [18]. 0 level means no visible collateral blood flow and 4 level means collateral blood flowing to the vascular bed in all ischemic regions completely within $2 \mathrm{~s}$.

\section{Statistical analysis}

All data were analyzed using SPSS software (SPSS 21.0 version package, USA). The data were expressed as $\overline{\mathrm{x}} \pm \mathrm{s}$ and further analyzed by $\mathrm{t}$ test and $\chi^{2}$ test. $\mathrm{P}<0.05$ was considered as statistically significant.

\section{Results}

Cerebral vessel stenotic degree was significantly different between the T2D group and the control group $\left(\chi^{2}=20.03\right.$, $\mathrm{P}<0.05)$. Patients with moderate to severe stenosis and occlusion were more in T2D group than those in the control group $\left(\chi^{2}=18.94, \mathrm{P}<0.05\right)$ (Table 2$)$. There were significant differences in the scope of lesion, diffuse vascular lesions and collateral compensative capacity between two groups $(\mathrm{P}<0.05$, Table 3). Although multiple vessel lesions were the main lesions, the incidence rate was higher obviously in T2D group (71.83\% vs. $53.46 \%)$ with diffuse stenoses (64.79\%) and no compensatory form collateral vessels $(71.13 \%)$. Control group was mainly with non-diffuse vascular lesions and compensatory form collateral vessels.

In 3 subgroup, with the increase of glycosylated hemoglobin (unsatisfactory blood glucose control), the incidence rate of moderate and severe stenosis was increasing significantly (Table 2). Along with unsatisfactory blood glucose control, the incidence rate of multiple and diffuse vessel lesion was markedly increased as well. What's more, the collateral compensative capacity was gradually diminished, which had a statistical significance in each group $(\mathrm{P}<0.05$, Table 3$)$.

Table 1. The general data of the patients.

\begin{tabular}{|c|c|c|c|c|c|c|c|c|}
\hline Group & $\mathbf{n}$ & $\begin{array}{l}\text { Gentle } \\
\text { (M/F) }\end{array}$ & $\begin{array}{l}\text { Age } \\
(\bar{x} \pm s)\end{array}$ & $\begin{array}{l}\text { Coronary disease } \\
\text { case }(\%)\end{array}$ & $\begin{array}{l}\text { Dysregulation } \\
\text { metabolism } \\
\text { case }(\%)\end{array}$ & of & lipid & $\begin{array}{l}\text { Hypertension } \\
(\%)\end{array}$ \\
\hline T2D & 152 & $69 / 83$ & $61.37 \pm 12.25$ & 98 (66.47) & 121 (79.61) & & & $101(66.45)$ \\
\hline Control & 184 & $97 / 87$ & $63.14 \pm 11.34$ & $104(56.52)$ & $116(63.04)$ & & & $125(67.93)$ \\
\hline$T$ or $x^{2}$ & & $1.50^{\#}$ & $1.37^{\#}$ & $1.88^{\#}$ & $10.20^{*}$ & & & $0.03^{\#}$ \\
\hline
\end{tabular}

Table 2. The lesion degree of angiography case (\%).

\begin{tabular}{lllll}
\hline Group & $\mathbf{n}$ & normal & mild & $\begin{array}{l}\text { Moderate, severe and } \\
\text { occlusion }\end{array}$ \\
\hline T2D & 152 & $10(6.58)$ & $19(12.50)$ & $123(80.92)$ \\
\hline Control & 184 & $25(13.59)$ & $52(28.26)$ & $107(58.15)$ \\
\hline X2 $^{2}$ & & $3.66^{\#}$ & $11.48^{*}$ & $18.94^{*}$ \\
\hline T2D-1 & 30 & $4(13.33)$ & $9(30.00)$ & $17(56.67)$ \\
\hline T2D-2 & 56 & $3(5.36)$ & $9(16.07)$ & $44(78.57)$ \\
\hline
\end{tabular}

\begin{tabular}{|c|c|c|c|c|}
\hline T2D-3 & 66 & $3(4.55)$ & $2(3.03)$ & $61(92.42)$ \\
\hline$x^{2}$ & \multicolumn{4}{|c|}{$x^{2}=17.91^{*}$} \\
\hline T2D-1: T2D-2 & & $x^{2}=0.77^{\#}$ & $x^{2}=1.53^{\#}$ & $x^{2}=3.55^{\#}$ \\
\hline T2D-2: T2D-3 & & $x^{2}=0.05^{\#}$ & $x^{2}=4.79^{*}$ & $x^{2}=3.76^{*}$ \\
\hline T2D-3: T2D-1 & & $x^{2}=1.24^{\#}$ & $x^{2}=12.25^{*}$ & $x^{2}=15.04^{*}$ \\
\hline
\end{tabular}

Table 3. The scope and degree of vascular lesion case (\%).

\begin{tabular}{|c|c|c|c|c|c|c|c|}
\hline \multirow[t]{2}{*}{ Group } & \multirow[t]{2}{*}{$\mathbf{n}$} & \multicolumn{2}{|c|}{ Scope } & \multicolumn{2}{|c|}{ Diffuse vascular lesions } & \multicolumn{2}{|c|}{ Collateral compensative capacity } \\
\hline & & Single & Multiple & Yes & No & With & Without \\
\hline T2D & 142 & $40(28.17)$ & $102(71.83)$ & $92(64.79)$ & $50(35.21)$ & $41(28.87)$ & $101(71.13)$ \\
\hline Control & 159 & $74(46.54)$ & $85(53.46)$ & $62(38.99)$ & $97(61.01)$ & $109(68.55)$ & $50(31.45)$ \\
\hline
\end{tabular}




\begin{tabular}{|c|c|c|c|c|c|c|c|}
\hline$x^{2}$ & & & $9.99^{*}$ & & $18.96^{*}$ & & $45.67^{*}$ \\
\hline T2D-1 & 30 & $16(53.33)$ & $14(46.67)$ & $12(40.00)$ & $18(60.00)$ & $17(56.67)$ & $13(43.33)$ \\
\hline T2D-2 & 56 & $17(30.36)$ & 39 (69.64) & $34(60.71)$ & $22(39.29)$ & $20(35.71)$ & $36(64.29)$ \\
\hline T2D-3 & 66 & $10(15.15)$ & $56(84.85)$ & $51(77.27)$ & $15(22.73)$ & $7(10.61)$ & 59 (89.39) \\
\hline$x^{2}$ & & $14.99^{*}$ & & $14.47^{*}$ & & $23.26^{*}$ & \\
\hline T2D-1: T2D-2 & & & $x^{2}=3.44^{\#}$ & & $x^{2}=2.59^{\#}$ & & $X^{2}=2.67^{\#}$ \\
\hline T2D-2: T2D-3 & & & $x^{2}=5.47^{*}$ & & $x^{2}=3.18^{\#}$ & & $x^{2}=9.67^{*}$ \\
\hline T2D-3: T2D-1 & & & $x^{2}=13.35^{*}$ & & $x^{2}=11.10^{*}$ & & $x^{2}=15.05^{*}$ \\
\hline
\end{tabular}

\section{Discussion}

TIA is the most important independent risk factor of cerebral infarction, and the recent frequency of TIA is a super alert which the risk of cerebral infarction is $4 \%-10 \%$ within 7 days and 10\%-20\% within 90 days [19,20]. The intracranial and extracranial artery stenosis may be the most important pathogenesis of TIA. If primary intracranial artery stenosis is greater than $50 \%$ in patients with ischemic cerebrovascular disease, the recurrence rate of cerebral infarction will be $19.0 \%$ in the next 1.8 years and $77 \%$ will occur within the original narrow blood vessel. If intracranial artery stenosis is more than $70 \%$, cerebral infarction will be more likely to recur [21]. Vermeet et al. found that in patients with TIA or mild cerebral infarction, the risk of cerebral infarction is 2 times higher in cases with abnormal glucose tolerance than cases with normal blood glucose, while the risk is almost 3 times in cases with diabetes [22]. In China, about $1 / 3$ ischemic cerebrovascular disease patients were accompanied with intracranial artery stenosis and diabetes, which diabetes is an important independent factor for intracranial arterial stenosis [23]. Our results in this study is in accord with Thomas who showed diabetic cerebral infarction patients often with multiple vessel stenosis by transcranial Doppler [24,25]. These mean cerebral artery lesions in TIA patients with T2D changed widely and more serious, which may be related to the abnormal blood lipid metabolism, changes in blood fibrinolysis, insulin resistance and high blood glucose. Lee et al. found that patients with diabetes are the most likely to have a combined lesion of the carotid artery and the intracranial artery in all the risk factors [26]. This illustrates artery stenosis level in TIA patients with diabetes is heavier with more diffuse lesions and multiple lesions, and worse collateral circulation, which will lead to perfusion decreasing, and then appearing TIA, even acute cerebral infarction.

When cerebral artery appears severe stenosis or occlusion, two levels of compensation will be provided like primary and secondary pathways. Primary pathway is circulus arteriosus cerebri (Willis) including carotid artery, anterior cerebral artery, posterior cerebral artery, anterior communicating artery and posterior communicating artery. And secondary pathway is consisted by leptomeningeal anastomosis, posterior pericallosal artery, brain compensatory compensation and so on. In general, the primary pathway plays a compensatory role in the early stage of ischemia, and the secondary pathway begins to compensate when the primary pathway is not developed or cannot provide and maintain normal blood flow. At this time, the regulation ability and perfusion of cerebral blood vessels were damaged. When the artery is narrow, the collateral circulation is insufficient, resulting in ischemic symptoms (such as TIA). Collateral circulation failure will lead to cerebral infarction [27]. High blood glucose make vascular structural protein and collagen protein appear non-enzymatic glycosylation, leading to the proliferation of endothelial cells in small arteries and capillaries, thickening of intima and basement membrane, causing serious intracranial arteriosclerosis and high blood viscosity. As a result, the rate of blood flow slowed down, affecting the establishment of collateral circulation.

\section{Conclusion}

This study found severe cerebral vascular stenosis is more easy to happen on patients of TIA accomplicated with T2D, which was distinguished by multiple vascular lesions, diffuse vascular lesions and less collateral compensative capacity. What's more, unsatisfactory blood glucose control can aggravate the above damages. Thus, risk factors of TIA such as diabetes should be paid enough attention. Closely monitoring blood glucose, strictly controlling blood glucose, reducing the incidence of diabetic complications, reducing and delaying cerebral blood supply artery lesions are still the key to current exploration.

\section{Acknowledgement}

All data and experiments were done by my team. Here we thank professor Zaiyu Guo for energetic support and help in the process of clinical work. I really very much appreciate your years of guidance and help.

\section{References}

1. Turner G, Calvert M, Feltham M, Ryan R, Marshall T. Fatigue, psychological and cognitive impairment following transient ischemic attack (TIA). North American Primary Care Research Group 2015. 
2. Easton J, Saver J, Albers G, Alberts M, Chaturvedi S, Feldmann E, Hatsukami T, Higashida R, Johnston S, Kidwell C, Lutsep H, Miller E, Sacco R. Definition and evaluation of transient ischemic attack: a scientific statement for healthcare professionals from the American Heart Association/American Stroke Association Stroke Council; Council on Cardiovascular Surgery and Anesthesia; Council on Cardiovascular Radiology and Intervention; Council on Cardiovascular Nursing; and the Interdisciplinary Council on Peripheral Vascular Disease. The American Academy of Neurology affirms the value of this statement as an educational tool for neurologists. Stroke 2009; 6: 2276-2293.

3. Ay H, Arsava EM, Johnston SC, Vangel M, Schwamm LH, Furie KL, Koroshetz WJ, Sorensen AG. Clinical and imaging-based prediction of stroke risk after transient ischemic attack: the CIP model. Stroke 2009; 1: 181-186.

4. Merwick A, Albers G, Amarenco P, Arsava E, Ay H, Calvet D, Coutts S, Cucchiara B, Demchuk A, Furie K, Giles M, Labreuche J, Lavallée P, Mas J, Olivot J, Purroy F, Rothwell P, Saver J, Sheehan O, Stack J, Walsh C, Kelly P. Addition of brain and carotid imaging to the $\mathrm{ABCD}^{2}$ score to identify patients at early risk of stroke after transient ischaemic attack: a multicentre observational study. Lancet Neurology 2010; 11: 1060-1069.

5. Santulli G. Thrombolysis outcomes in acute ischemic stroke patients with prior stroke and diabetes mellitus. Neurology 2012; 78: 840.

6. Ritter L, Davidson L, Henry M, Davis-Gorman G, Morrison H, Frye JB, Cohen Z, Chandler S, McDonagh P, Funk JL. Exaggerated Neutrophil-Mediated Reperfusion Injury after Ischemic Stroke in a Rodent Model of Type 2 Diabetes. Microcirculation 2011; 7: 552-561.

7. Ataoglu HE, Saler T, Uzunhasan I, Yenigun M, Yigit Z, Temiz LU, Saglam Z, Cetin F, Kumbasar B, Sar F. Additional value of carotid artery intima-media thickness in cardiovascular risk assessment by Framingham risk-score in Type 2 diabetes: a retrospective cohort study. Journal of Diabetes 2009; 3: 188-193.

8. Turan T, Makki A, Tsappidi S, Cotsonis G, Lynn M, Cloft H, Chimowitz M. Risk factors associated with severity and location of intracranial arterial stenosis. Stroke 2010; 8: 1636-1640.

9. Taylor RA, Weigele JB, Kasner SE. Current management of symptomatic intracranial stenosis. Curr Atheroscler Rep 2011; 4: 321-329.

10. Suwanwela N,Chutinetr A. Risk factors for atherosclerosis of cervicocerebral arteries: intracranial versus extracranial. Neuroepidemiol 2003; 1: 37-40.

11. Parsian A, Ramezani M, Ghadimi N. A hybrid neural network-gray wolf optimization algorithm for melanoma detection. Biomed Res 2017; 28: 3408-3411.

12. Chilcote W, Modic M, Pavlicek W, Little J, Furlan A, Duchesneau P, Weinstein M. Digital subtraction angiography of the carotid arteries: a comparative study in 100 patients. Radiology 1981; 2: 287-295.
13. Ebrahimian H. Distributed Diode Single-Balanced Mixer Using Defected and Protruded Structures for Doppler Radar Applications. Applied Comput Electromag Soc J 2015.

14. Razmjooy N, Ramezani M, Ghadimi N. Imperialist competitive algorithm-based optimization of neuro-fuzzy system parameters for automatic red-eye removal. Int $\mathrm{J}$ Fuzzy Syst 2017; 11: 1-13.

15. Organization WH. Definition, diagnosis and classification of diabetes mellitus and its complications: Report of a WHO Consultation. Part 1: Diagnosis and Classification of Diabetes Mellitus. Geneva, World Health Org, 1999.

16. Rnett H. North American symptomatic carotid endarterectomy trial, methods, patient characteristics, and progress. Stroke 1991; 6: 711-720.

17. Iqbal M, Bilal S, Sarwar S, Counihan T. Is it important to classify ischaemic stroke? Irish Med J 2012; 2: 52-54.

18. Higashida R, Furlan A. Trial design and reporting standards for intra-arterial cerebral thrombolysis for acute ischemic stroke. Stroke 2003; 8: e109-e137.

19. Giles M, Rothwell P. Risk of stroke early after transient ischaemic attack: a systematic review and meta-analysis. Lancet Neurol 2007; 12: 1063-1072.

20. Johnston S, Rothwell P, Nguyen-Huynh M, Giles M, Elkins J, Bernstein A, Sidney S. Validation and refinement of scores to predict very early stroke risk after transient ischaemic attack. Lancet 2007; 9558: 283-295.

21. Di Carlo A, Lamassa M, Baldereschia M, Pracucci G, Consoli D, Wolfe CDA, Giroud M, Rudd A, Burger I, Ghetti A, Inzitar D. Risk factors and outcome of subtypes of ischemic stroke. Data from a multicenter multinational hospital-based registry. The European Community Stroke Project. J Neurol Sci 2006; 1: 143-150.

22. Vermeer SE, Sandee W, Algra A, Koudstaal PJ, Kappelle LJ, Dippel DW; Dutch TIA Trial Study Group. Impaired glucose tolerance increases stroke risk in nondiabetic patients with transient ischemic attack or minor ischemic stroke. Stroke 2006; 37: 1413-1417.

23. Wong K, Li H, Chan Y, Ahuja A, Lam W, Wong A, Kay R, Albers G. Use of transcranial Doppler ultrasound to predict outcome in patients with intracranial large-artery occlusive disease. Stroke 2000; 11: 2641-2647.

24. Ghadimi N, Ojaroudi M. A novel design of low power rectenna for wireless sensor and RFID applications. Wireless Personal Commun 2014; 2: 1177-1186.

25. Thomas G, Lin J, Lam W. Increasing severity of cardiovascular risk factorrs with increosing middle cerebral artery stenotic involvement in type 2 diabetic Chinese patients with asymptomatic cerebrovascular. Diabetes Cart 2004; 1121-1126.

26. Lee S, Cho S, Moon H, Shon Y, Lee K, Kim D, Lee B, Byun H, Han S, Chung C, Albers G. Combined extracranial and intracranial atherosclerosis in Korean patients. Arch Neurol 2003; 11: 1561-1564.

27. Liebeskind D, Cotsonis GA, Saver JL, Lynn MJ, Cloft HJ, Chimowitz MI. Collateral circulation in symptomatic 
intracranial atherosclerosis. J Cerebral Blood Flow Metabol 2011; 5: 1293-1301.

\section{*Correspondence to}

Zaiyu Guo

Department of Neurology

Tianjin Teda Hospital

PR China 